PROCEEDINGS OF THE

AMERICAN MATHEMATICAL SOCIETY

Volume 128, Number 9, Pages 2637-2640

S 0002-9939(00)05276-X

Article electronically published on February 28, 2000

\title{
LIOUVILLE NUMBERS, RAJCHMAN MEASURES, AND SMALL CANTOR SETS
}

\author{
CHRISTIAN E. BLUHM
}

(Communicated by Christopher D. Sogge)

\begin{abstract}
We show that the set of Liouville numbers carries a positive measure whose Fourier transform vanishes at infinity. The proof is based on a new construction of a Cantor set of Hausdorff dimension zero supporting such a measure.
\end{abstract}

\section{INTRODUCTION}

In the year 1844 JosePH LiOUVILLE constructed an interesting class of transcendental numbers, namely

$$
\mathbb{L}=\left\{x \in \mathbb{R} \backslash \mathbb{Q}: \forall n \in \mathbb{N}: \exists q \in \mathbb{N}:\|q x\|<q^{-n}\right\},
$$

now called the set of Liouville numbers. Here $\|x\|=\min _{m \in \mathbb{Z}}|x-m|$ denotes the distance of a real number $x$ to the nearest integer. For example, the number $\sum 10^{-k !}=0.110001000 \ldots$ (where the 1 is only in places $n$ !) belongs to $\mathbb{L}$. From the well known theorem of JARNIK [4 and BESICOVITCH 1 it follows immediately that $\mathbb{L}$ has Hausdorff dimension zero, so we consider $\mathbb{L}$ to be a 'small' set.

In this note we show that $\mathbb{L}$ supports a positive measure whose Fourier transform vanishes at infinity. Such measures are called Rajchman measures; see the survey article by Lyons [6] for references. A detailed discussion of related constructions can be found in KöRNER's paper [5].

The proof of our result is based on a new construction of a Cantor set with Hausdorff dimension zero carrying a Rajchman measure.

\section{MAin RESUlts}

In the sequel $\mathbb{P}_{M}$ denotes the set of prime numbers between $M$ and $2 M$ where $M$ is a positive integer. We choose a sequence of positive integers $\left(M_{k}\right)_{k \in \mathbb{N}}$ with $M_{1}<2 M_{1}<M_{2}<2 M_{2}<M_{3}<2 M_{3}<\cdots$ and define the set

$$
S_{\infty}=\bigcap_{k=1}^{\infty} \bigcup_{p \in \mathbb{P}_{M_{k}}}\left\{x \in[0,1]:\|p x\| \leq p^{-1-k}\right\} .
$$

Received by the editors September 1, 1998 and, in revised form, October 19, 1998.

1991 Mathematics Subject Classification. Primary 42A38; Secondary 28A80.

Key words and phrases. Liouville numbers, Rajchman measure, Cantor set.

(C)2000 American Mathematical Society 
In fact, $S_{\infty}$ is compact because $\bar{E}_{k}(p)=\left\{x \in[0,1]:\|p x\| \leq p^{-1-k}\right\}$ equals

$$
\left[0, p^{-2-k}\right] \cup \bigcup_{m=1}^{p-1}\left[\frac{m}{p}-p^{-2-k}, \frac{m}{p}+p^{-2-k}\right] \cup\left[1-p^{-2-k}, 1\right] .
$$

Proposition 2.1. $S_{\infty}$ is a Cantor set of Hausdorff dimension zero.

Proof. According to (2.1) the set $\bar{E}_{k}(p)$ can be covered by $p+1$ intervals of length $\leq 2 p^{-2-k}$. For every $k \in \mathbb{N}$ and $\alpha=3 /(2+k)$ this implies

$$
H^{\alpha}\left(S_{\infty}\right) \leq \sum_{p \in \mathbb{P}_{M_{k}}}(p+1)\left(2 p^{-2-k}\right)^{3 /(2+k)}<\infty .
$$

Therefore, $S_{\infty}$ has Hausdorff dimension zero.

For the following theorem recall that the Fourier transform of a positive bounded measure $\mu$ is defined by

$$
\hat{\mu}(x)=\int_{\mathbb{R}} e^{-2 \pi i x t} d \mu(t) \quad(x \in \mathbb{R}) .
$$

Theorem 2.2. There exists a sequence $\left(M_{k}\right)_{k \in \mathbb{N}}$ such that the corresponding set $S_{\infty}$ supports a positive measure $\mu_{\infty}$ with

$$
\lim _{|x| \rightarrow \infty} \hat{\mu}_{\infty}(x)=0 .
$$

As an application we obtain

Theorem 2.3. The set $S_{\infty} \backslash \mathbb{Q}$ is contained in $\mathbb{L}$. Therefore, the set of Liouville numbers carries a Rajchman measure.

Proof. From the definition of $\mathbb{L}$ and $S_{\infty}$ it is obvious that $S_{\infty} \backslash \mathbb{Q} \subset \mathbb{L}$. The Rajchman measure $\mu_{\infty}$ is supported by $S_{\infty}$. Removing the rational points from $S_{\infty}$ means removing a zero set from the support of $\mu_{\infty}$. By a simple regularity argument we can replace $\operatorname{supp}\left(\mu_{\infty}\right) \backslash \mathbb{Q}$ by a compact set with positive $\mu_{\infty}$-measure.

\section{Proof of Theorem 2.2}

Proof. The proof of Theorem 2.2 is based on a modification of a construction elaborated in [2]. There (in section 3) we constructed, for given positive $\alpha>0$ and positive integers $M$, certain 1-periodic functions $g_{M} \in C^{2}(\mathbb{R})$ with

$$
\operatorname{supp}\left(g_{M}\right) \subseteq \bigcup_{p \in \mathbb{P}_{M}}\left\{x:\|p x\| \leq p^{-1-\alpha}\right\} \quad \text { and } \quad \hat{g}_{M}(0)=1 .
$$

Moreover, these functions $g_{M}$ had the following nice property ([2], Lemma 3.2):

Lemma 3.1. For every $\psi \in C_{c}^{2}(\mathbb{R})$ and $\delta>0$ there exists $M_{0}=M_{0}(\psi, \delta)$ s.t.

$$
\left|\left[\psi g_{M}\right]^{\wedge}(x)-\hat{\psi}(x)\right| \leq \delta \cdot \theta(x) \quad \forall x \in \mathbb{R}
$$

for all $M \geq M_{0}$, where $\theta(x)=(1+|x|)^{-1 /(2+\alpha)} \cdot \log (e+|x|) \cdot \log (e+\log (e+|x|))$.

We will apply Lemma 3.1 to our situation by replacing $\alpha$ by $k$ and $\theta$ by

$$
\theta_{k}(x)=(1+|x|)^{-1 /(2+k)} \cdot \log (e+|x|) \cdot \log (e+\log (e+|x|)) \quad(k \in \mathbb{N}) .
$$


To be more precise, we fix an initial function $\psi_{0}: \mathbb{R}_{+} \rightarrow \mathbb{R}_{+}$with

$$
\psi_{0} \in C_{c}^{2}(\mathbb{R}), \quad \int \psi_{0}(x) d x=1,\left.\quad \psi_{0}\right|_{] 0,1[}>0, \quad \text { and }\left.\quad \psi_{0}\right|_{\mathbb{R} \backslash[0,1]} \equiv 0 .
$$

Next we define a sequence $\left(\tau_{k}\right)_{k \in \mathbb{N}}$ by $\tau_{k}=\left(\max _{x \in \mathbb{R}} \theta_{k}(x)\right)^{-1}$. As a first step we replace $\alpha$ in the setting above by $k=1$. According to Lemma 3.1 we can find a positive integer $M_{1}=M_{1}\left(\psi_{0}, \tau_{1} 3^{-1}\right)$ such that

$$
\left|\left[\psi g_{M_{1}}\right]^{\wedge}(x)-\hat{\psi}(x)\right| \leq \tau_{1} 3^{-1} \theta_{1}(x) \quad \forall x \in \mathbb{R} .
$$

Now we repeat the same procedure, but this time replacing $\alpha$ by $k=2, \theta_{1}$ by $\theta_{2}$, and $\psi$ by $\psi_{0} g_{M_{1}}$. Then again Lemma 3.1 implies the existence of an integer $M_{2}=M_{2}\left(\psi_{0} g_{M_{1}}, \tau_{2} 3^{-2}\right)$ such that

$$
\left|\left[\psi g_{M_{1}} g_{M_{2}}\right]^{\wedge}(x)-\left[\psi_{0} g_{M_{1}}\right]^{\wedge}(x)\right| \leq \tau_{2} 3^{-2} \theta_{2}(x) \quad \forall x \in \mathbb{R} .
$$

By repeating this process we obtain for every index $k$ an integer

$$
M_{k}=M_{k}\left(\psi_{0} g_{M_{1}} g_{M_{2}} \cdots g_{M_{k-1}}, \tau_{k} 3^{-k}\right) \quad(k \in \mathbb{N}),
$$

fulfilling the corresponding estimation.

Now we assume $S_{\infty}$ to be constructed according to $\left(M_{k}\right)_{k \in \mathbb{N}}$. We set

$$
G_{0}=1, \quad \text { and } \quad G_{k}=g_{M_{1}} \cdots g_{M_{k}} \quad(k \in \mathbb{N}) .
$$

By Lemma 3.1 we obtain for every $k \in \mathbb{N}_{0}$ and all $x \in \mathbb{R}$

$$
\left|\left[\psi_{0} G_{k+1}\right]^{\wedge}(x)-\left[\psi_{0} G_{k}\right]^{\wedge}(x)\right| \leq \tau_{k+1} 3^{-k-1} \theta_{k+1}(x) .
$$

Let $\lambda$ be Lebesgue measure and define a sequence of measures by

$$
\mu_{k}=\psi_{0} G_{k} \lambda \quad\left(k \in \mathbb{N}_{0}\right) .
$$

Because of (3.2) the sequence $\left(\hat{\mu}_{k}\right)_{k \in \mathbb{N}_{0}}$ is a Cauchy sequence w.r.t. the supremum norm. Taking $\hat{g}_{M_{k}}(0)=1$ into account (see (3.1)), we conclude the weak convergence of $\left(\mu_{k}\right)_{k}$ to a bounded measure $\mu_{\infty}$ (Lévy's continuity theorem). Moreover, by (3.2) and a geometric series estimate we get $\left|\hat{\mu}_{\infty}(0)-\hat{\psi}(0)\right| \leq \frac{1}{2}$, so that $\mu_{\infty}$ has at least mass $\frac{1}{2}$. The claimed Fourier asymptotic of $\mu_{\infty}$ follows easily from (3.2) and a simple geometric series argument, also taking into account that $\hat{\mu}_{p}(x)=O\left(|x|^{-2}\right)$ for fixed $p$. The construction of $\mu_{\infty}$ is based on successive multiplication of densities $g_{M_{k}}$. Therefore, by (3.1) it is clear that the support of $\mu_{\infty}$ must be contained in the Cantor set $S_{\infty}$. This concludes the proof of Theorem 2.2

Remark 3.2. Why prime numbers? Let us sketch the answer. The proof of Lemma 3.1 ([2], section 4) rests on the prime number theorem $\# \mathbb{P}_{M} \sim M / \log M$ (HARDY AND WRIght [3] (22.19.3)). So it is clear that although the number of primes between $M$ and $2 M$ is strictly increasing with $M$, the primes are somehow 'thinning out' at infinity. This observation is of great importance in the proof of Lemma 3.1 when one tries to allow the ' $\delta$ ' in the estimation to become arbitrarily small.

\section{REFERENCES}

[1] Besicovitch, A. S., Sets of fractional dimensions (IV): on rational approximation to real numbers, J. Lond. Math. Soc. 9 (1934), 126-131

[2] Bluhm, C., On a theorem of Kaufman: Cantor-type construction of linear fractal Salem sets, Ark. Mat. 36 (1998), 307-316 MR 99i:43009

[3] Hardy, G. H., Wright, E. M., An introduction to the theory of numbers, Oxford University Press, $4^{\text {th }}$ ed. (1971) MR 81i:10002 (5th edition) 
[4] Jarnik, V., Zur metrischen Theorie der diophantischen Approximation, Prace Mat.-Fiz. 36 (1928/29), 91-106

[5] Körner, T. W., On the theorem of Ivashev-Musatov III, Proc. London Math. Soc. (3) 53 (1986), 143-192 MR 88f:42021

[6] Lyons, R., Seventy Years of Rajchman Measures, J. Fourier Anal. Appl., Kahane Special Issue (1995), 363-377 MR 97b:42019

Department of Mathematics, University of Greifswald, Jahnstrasse 15A, D-17487 Greifswald, Germany

E-mail address: bluhm@rz.uni-greifswald.de 